\title{
Span, $\mathrm{CRUNCH}$, and Beyond: Working Memory Capacity and the Aging Brain
}

\author{
Nils J. Schneider-Garces, Brian A. Gordon, Carrie R. Brumback-Peltz, Eunsam Shin, \\ Yukyung Lee, Bradley P. Sutton, Edward L. Maclin, Gabriele Gratton, and Monica Fabiani \\ University of Illinois at Urbana-Champaign
}

\begin{abstract}
Neuroimaging data emphasize that older adults often show greater extent of brain activation than younger adults for similar objective levels of difficulty. A possible interpretation of this finding is that older adults need to recruit neuronal resources at lower loads than younger adults, leaving no resources for higher loads, and thus leading to performance decrements [Compensation-Related Utilization of Neural Circuits Hypothesis; e.g., Reuter-Lorenz, P. A., \& Cappell, K. A. Neurocognitive aging and the compensation hypothesis. Current Directions in Psychological Science, 17, 177-182, 2008]. The Compensation-Related Utilization of Neural Circuits Hypothesis leads to the prediction that activation differences between younger and older adults should disappear when task difficulty is made subjectively comparable. In a Sternberg memory search task, this can be achieved by assessing brain activity as a function of load relative to the individual's memory span, which declines with age. Specifically, we hypothesized a nonlinear relationship between load and both performance and brain activity and predicted that asymptotes in the brain activation function should correlate with performance asymptotes (corresponding to working memory span). The results suggest that age differences in brain activation can be largely attributed to individual variations in working memory span. Interestingly, the brain activation data show a sigmoid relationship with load. Results are discussed in terms of Cowan's [Cowan, N. The magical number 4 in short-term memory: A reconsideration of mental storage capacity. Behavioral and Brain Sciences, 24, 87-114, 2001] model of working memory and theories of impaired inhibitory processes in aging.
\end{abstract}

\section{INTRODUCTION}

Working memory (WM) is a system that allows us to store and manipulate small amounts of information for a short time (Baddeley, 1986; Baddeley \& Hitch, 1974). One of the most intriguing findings in cognitive psychology is that the capacity of WM is in fact very limited, although there is some debate as to exactly how many items can be maintained and manipulated. Miller (1956), in a classic article, proposed that the capacity of WM is $7 \pm 2$ items. However, in a more recent review of a large number of studies, Cowan (2001) proposed that the core of the WM system can only hold $4 \pm 1$ items and that additional processes such as "chunking" are required for more items. An important aspect of Cowan's model is that WM is seen as a part of a more extended memory system, in which a small number of items are activated out of a much larger pool, so as to be readily available for the performance of a particular task. The limitation, therefore, is not really in memory capacity per se but in how many items can be kept into the focus of attention at any point in time. Thus, this "activation capacity" is assumed to be dependent on attention deployment, and WM is assumed to be limited by attention span. A similar view has been proposed by Engle

Reprint requests should be sent to Monica Fabiani, Beckman Institute, University of Illinois, 405 North Mathews Avenue, Urbana, IL 61801, or via mfabiani@illinois.edu. 
and Kane (2004), Kane, Bleckley, Conway, and Engle (2001), and Kane and Engle (2000). A related question is how WM capacity is linked to brain activations during WM tasks. To address this question, this study aims at examining in detail the changes in brain activity that are observed when WM capacity limits are reached.

Although the majority of studies of WM capacity have been carried out in young adults, in the last several decades researchers have also investigated how WM changes with age (e.g., Craik \& Byrd, 1982; Craik, 1968). Several studies have shown that, similarly to other cognitive functions, WM performance declines with increasing age (e.g., Bopp \& Verhaeghen, 2005; Park et al., 2002; Verhaeghen \& Salthouse, 1997). Several theories have been developed to explain this decline. For example, Salthouse (1996) proposed that aging leads to reduced speed of processing, rendering it more difficult to maintain many items in memory at a time. However, it is also possible to link age-related WM decline to a reduced ability to maintain an appropriate/stable attention focus. In fact, there is substantial evidence of reduced inhibition of the processing of distracting or irrelevant information in older adults, which may support such a scenario (Hasher, Lustig, \& Zacks, 2008; Hasher \& Zacks, 1988). In our own research, we have also found evidence supporting the claim that older adults may have problems inhibiting the processing of irrelevant information present within the experimental context (Fabiani, Low, Wee, Sable, \& Gratton, 2006; Fabiani, Friedman, \& Cheng, 1998; Fabiani \& Friedman, 1995). An inappropriate focus on irrelevant/distracting information, stemming from age-related difficulties in attention control, may effectively reduce the WM capacity that is available for the task at hand and lead to decreased performance.

Interestingly, this view of WM decline in aging does not necessarily imply that older adults should show down-regulation of their brain activity during WM tasks compared with younger adults. Indeed, functional neuroimaging data provide numerous examples of an increased number of areas showing up-regulation during the performance of several cognitive tasks, including WM tasks (e.g., Riecker et al., 2006; Park et al., 2003; ReuterLorenz, Stanczak, \& Miller, 1999; Grady et al., 1994; for a review, see Reuter-Lorenz \& Lustig, 2005). In many cases, the data indicate the occurrence of bilateral activations in older adults when younger adults only show unilateral activity (Hemispheric Asymmetries Are Reduced in OLD, HAROLD); Cabeza et al., 2004; Cabeza, 2002; see also ReuterLorenz et al., 1999). In other studies, this up-regulation has involved areas within the same hemisphere (e.g., Payer et al., 2006).

These age-related increases in brain activity are consistent with the concept of dedifferentiation (Lindenberger \& Baltes, 1997; see also Spearman, 1927): Older adults may not be able to activate networks as selectively or as efficiently as younger adults, therefore activating networks in both hemispheres or involving additional areas. Thus, such data could be interpreted as indicators of neuronal dysfunction (i.e., the inability to suppress inappropriate processing leading to conflict or reduced availability of resources; for example, Zarahn, Rakitin, Abela, Flynn, \& Stern, 2007; Rypma, Berger, \& D'Esposito, 2002) or as compensatory activity for impaired functioning (i.e., a vicarious processing route may be used when the appropriate processing units are not as readily available; for example, Cabeza, 2002; Reuter-Lorenz, Marshuetz, Jonides, \& Smith, 2001; Rypma \& D'Esposito, 2001; McIntosh et al., 1999).

Recent studies show that increasing task loads may induce not only older adults but even younger adults to up-regulate activity in some cortical regions (e.g., Mattay et al., 2006). To account for both the age-related deficits and these load effects, Reuter-Lorenz and Cappell (2008) and Reuter-Lorenz and Lustig (2005) proposed that, in general, people will activate more cortical regions as task load increases (Compensation-Related Utilization of Neural 
Circuits Hypothesis; CRUNCH). However, because of less efficient processing, it may be necessary for older adults to recruit these regions at lower load levels than younger adults. This hypothesis thus argues that older adults might recruit cognitive resources at lower loads to compensate for cognitive decline. Therefore, one would expect to see a sharper increase in fMRI signal for low load levels in older adults than in younger adults.

How would this hypothesis interact with the capacity limits of WM? We hypothesize that, as WM load increases, brain activity should increase up to where the memory capacity limit is reached. After that, brain activity should stop increasing, either because there are no further resources available or because there is no performance advantage in deploying brain resources any further. However, this limit should be reached earlier in older adults than in younger adults, resulting in a ceiling effect for both the fMRI signal and performance. If load is varied parametrically across several levels from low to high, older adults' fMRI activation should follow a nonlinear pattern, with a sharp increase at the beginning and a flattening at higher loads. The predicted fMRI asymptote at higher loads corresponds to the performance pattern predicted by the Cowan's (2001) theory; CRUNCH adds the prediction of a sharper increase in fMRI signal for low loads in older adults. Observation of both patterns requires data from several levels at both low and high loads.

The Sternberg (1966) memory search task appears to be a particularly useful tool to examine the relationship between memory load and brain activation in younger and older adults. This paradigm allows for parametric variations of memory load by using different memory set sizes. It has also been extensively studied using fMRI (Veltman, Rombouts, \& Dolan, 2003; Bunge, Ochsner, Desmond, Glover, \& Gabrieli, 2001; D'Esposito, Postle, \& Rypma, 2000; Henson, Burgess, \& Frith, 2000), including experiments comparing the results obtained in younger and older adults (e.g., Zarahn et al., 2007; Rypma et al., 2002). These data generally show increasing activations in medial and lateral $\mathrm{pFC}$ as well as in parietal cortex, with increasing memory loads. Results have also indicated the existence of age-related changes, but the interpretation of these effects has been complicated by the presence of large individual differences (Rypma, Berger, Genova, Rebbechi, \& D'Esposito, 2005), which have been attributed to variations in strategies. These strategic differences may have been in part due to the use of a slow event-related design, with very long intervals (>10 sec) between the presentation of the memory set and of the probe stimulus, which may have encouraged participants to use elaborative rehearsal. In the current study, we parametrically varied memory set size from 2 to 6 and chose a much shorter interval $(4 \mathrm{sec})$, which should reduce strategic differences, but still leave enough time for both younger and older adults to encode the memory set stimuli.

When considered in all its implications, the CRUNCH model explains overrecruitment and underrecruitment of brain areas in older adults in terms of the relative activation necessary to cope with the task and to compensate for deficits. Taken in this light, therefore, $\mathrm{CRUNCH}$ leads to a very strong prediction: The difference in brain activation level between younger and older subjects should disappear once the difficulty of the task is equated between the two groups. This should occur when memory load is not considered in absolute terms but relative to WM span. In turn, this requires the assessment of memory capacity/span in each individual, so that a curve of brain activation by subjective memory load can be computed on a subject-by-subject basis and then examined across groups. There are in fact several procedures that are commonly used to assess memory span. Of these, two of the most frequent are backward digit span (Wechsler, 1981) and operation span (O-SPAN; Engle \& Kane, 2004; Kane et al., 2001; Kane \& Engle, 2000). Although very useful, these measures are limited because they do not directly estimate WM capacity within the same task used to assess brain activity (in this case, the Sternberg task). It is therefore difficult to exactly scale 
the scores obtained by each individual subject in these span tests so that they are made consistent with the memory loads used in the Sternberg task.

To address this problem, we derived a measure of WM span directly from the performance obtained within the Sternberg task. We followed suggestions by Cowan (2001) to estimate the amount of information that is transmitted during the memory task (which we refer to here as "throughput"). We then used this measure to estimate the memory span of each individual within the Sternberg task, providing estimates that are expressed in the same unit (memory load as a function of the number of items in the memory set) used to classify the brain activation function. This allowed us to measure brain activity as a function of how large the memory load was with respect to memory span for each individual (for a similar approach in young adults see Todd \& Marois, 2005). Using these data, we could then evaluate whether similar activation by subjective load functions were found in younger and older adults—or, in other words, whether age-related differences in these functions disappeared when difficulty was normalized by WM capacity, as predicted by CRUNCH.

\section{METHODS}

\section{Participants}

This study was part of a more extended project aimed at examining changes in neurovascular coupling as a function of aging and physical fitness. For this reason, the older group was larger than the younger group. The original sample included 17 younger adults recruited from the University of Illinois' student population and 33 older adults recruited through ads in local newspapers, campus-wide e-mailings, and postings at area gyms, retirement homes, and community centers. For the purposes of the current study, however, the relevant measures were only available from a smaller set of 42 subjects (behavioral measures were not available in 4 subjects, and 4 additional subjects were discarded because of significant movement artifacts in fMRI recordings). Thus, the younger sample included 12 subjects (age range $=18-27$ years, mean age $=23.8$ years, 6 women); the older sample included 30 subjects (age range $=65-80$, mean age $=70.9$ years, 13 women). Younger and older adults did not differ in years of education or scores in the Vocabulary subtest of the Wechsler Adult Intelligence Scale-Revised (Wechsler, 1981). They were significantly different on the modified Mini-Mental Status examination (Mayeux, Stern, Rosen, \& Leventhal, 1981) and on the O-SPAN (La Pointe \& Engle, 1990). The demographic characteristics of the participants are summarized in Table 1.

Screening Procedures-Participants were screened based on a number of health and cognitive criteria. Prospective subjects were excluded from the study if they regularly took medications that are known to affect the CNS (e.g., beta blockers, CNS stimulants, antidepressants, antipsychotics, sedating antihistamines, or migraine medications). Subjects with serious or chronic medical conditions were also excluded. Additionally, subjects had to score at least 51 on the modified Mini-Mental Status examination, show no signs of depression on Beck's Depression Scale (Beck \& Steer, 1996), and score above or within one standard deviation of the average score for their age group on the Vocabulary subtest of the Wechsler Adult Intelligence Scale-Revised (Wechsler, 1981). All participants were righthanded (as assessed by the Edinburgh Handedness Inventory; Oldfield, 1971) and had normal or corrected-to-normal vision.

\section{Memory Paradigm and Procedures}

We used a modified version of Sternberg's memory search task (Sternberg, 1966), with memory set sizes two through six (see Figure 1). The stimuli to be encoded were uppercase letters (B, D, F, G, H, J, M, R, and T). To prevent a direct visual match, their corresponding 
lowercase letters were used as probes (see Bunge et al., 2001). The letters were selected because of their different shapes when presented in upper and lower case. Each letter subtended approximately $1.4^{\circ}$ of visual angle in the diagonal and was presented using a Resonance Technologies goggles system (Resonance Technologies, Northridge, CA).

Each trial was initiated by the presentation of a memory set comprising two to six uppercase letters presented simultaneously for $3 \mathrm{sec}$, followed by a screen containing only a fixation cross presented for $1 \mathrm{sec}$. After that, the probe was presented for $500 \mathrm{msec}$, followed by another fixation cross presented for $1.5 \mathrm{sec}$. During this 2-sec interval, participants had to indicate whether the probe was part of the preceding memory set by pressing the right or the left button on a response box with the corresponding hand. The response-hand assignments were counterbalanced across subjects. Each memory set was composed of letters chosen randomly from the set of letters listed above, with the proviso that no identical letters were allowed within the same memory set. The probe was part of the memory set on $50 \%$ of the trials. Five memory set size conditions $(2,3,4,5$, and 6$)$ were used in a blocked fashion and were presented in either ascending (2-6) or descending (6-2) order, counterbalanced across subjects. Set Sizes 2-6 were chosen because they encompass the memory span predicted by Cowan (2001). For each set size condition, a run consisted of four blocks of eight trials each, with a 20-sec fixation period before the first block and between each block. This yielded a total of four task blocks (32 trials) and four rest blocks per set size condition.

All subjects underwent a training session with 128 trials using Set Sizes 4-6 before being tested inside the MRI scanner. Further, a short training block with approximately 32 trials using Set Size 4 was administered just before the fMRI recording began to ensure that participants remembered the task instructions.

\section{Data Acquisition and Preprocessing}

The MRI data were recorded with a Siemens Allegra 3-T head-only scanner. The fMRI data were recorded with a fast echo-planar protocol (repetition time $=2 \mathrm{sec}$, echo time $=25$ msec, flip angle $=80^{\circ}$ ). Thirty-eight slices (3-mm thickness, 3-mm in-plane resolution, 0.3$\mathrm{mm}$ gap) were collected interleaved and parallel to the anterior and posterior commissures. A high-resolution T1-weighted MPRAGE (192 slices, $1 \times 1 \times 1 \mathrm{~mm}$ ) was also recorded to enable accurate anatomical coregistration. Finally, a fast T2-weighted image was also collected for coregistering the T2* image used for fMRI with the T1 image used for anatomical analysis.

The neuroimaging data were preprocessed and analyzed using FSL version 3.1 (http:// www.fmrib.ox.ac.uk/fsl/). Structural images were processed with SUSAN (part of FSL) to improve the signal-to-noise ratio and BET (part of FSL) was used to perform skull stripping. BET was also used on the functional images. In addition, the functional images were slicetime corrected, motion corrected using MCFLIRT, temporally filtered with a Gaussian highpass cutoff of $70 \mathrm{sec}$, and spatially smoothed with a 6-mm FWHM three-dimensional Gaussian kernel. Functional and structural images were coregistered and transformed into the Montreal Neurological Institute coordinates before group analyses were carried out.

\section{Data Analysis}

Behavior-The behavioral data (RT and accuracy) were analyzed with mixed-design ANOVAs with one between-subjects factor (Age) and one within-subjects factor (Set Size). For the ANOVA, the accuracy data were first transformed using the Fisher logit approximation to avoid ceiling effects. Note that, due to the use of a blocked fMRI design, we collapsed across probe items requiring yes or no responses for all these analyses. Further, no significant differences were found between descending and ascending set size 
presentation orders, so the data were combined for all behavioral and neuroimaging analyses.

In addition, we also estimated the amount of information transmitted (throughput), given the number of items in the memory set. Throughput is derived according to the following formula, which is mathematically identical to the $k$ formula introduced by Cowan (2001; see also Cowan et al., 2005):

$$
\text { Throughput }=\frac{\mathrm{ACC}-0.5}{0.5} * N \text { items, }
$$

where the chance level of 0.5 is subtracted from the uncorrected overall accuracy (ACC), then range corrected by dividing by 0.5 (as above-chance accuracy can only vary between 0.5 and 1) and finally multiplied by the number of items included in the memory set for that condition. This formula corrects for chance level and takes into account that more information is available at higher load levels. Note that if accuracy is 1 (perfect), the throughput is equal to the number of items in the memory set, which would indicate that all information available is processed (ideal function in Figure 3). By measuring throughput across increasing set sizes, we will be able to estimate WM capacity as the maximum amount of information transmitted across set sizes.

fMRI-The statistical analysis of fMRI data was carried out using FEAT (fMRI Expert Analysis Tool) Version 5.63, part of FSL (FMRIB's Software Library, www.fmrib.ox.ac.uk/ fsl). Group-level analyses were carried out using FLAME (FMRIB's Local Analysis of Mixed Effects) Stage 1 only (i.e., without the final MCMC-based stage; Woolrich, Behrens, Beckmann, Jenkinson, \& Smith, 2004; Beckmann, Jenkinson, \& Smith, 2003). The overall mean of each group was thresholded using clusters determined by $Z>5.0$ and a (corrected) cluster significance threshold of $p=.05$ (Worsley, Evans, Marrett, \& Neelin, 1992). ${ }^{1}$ A linear trend analysis was applied to the group-level analysis, separately for Set Sizes 2-4 and 4-6. Resulting $Z$ (Gaussianized $T / F$ ) statistic images were thresholded using clusters determined by $Z>3.1$ and a (corrected) cluster significance threshold of $p=.05$ (Worsley et al., 1992).

To determine differences in MR activity by set size slopes between younger and older adults, a peak voxel analysis was performed. ${ }^{2}$ The voxel showing the largest $Z$-score within each of a series of ROIs was selected for each subject and condition. The ROIs were drawn according to Brodmann's areas (BA) as implemented in the WFU Pickatlas (http:// www.fmri.wfubmc.edu; Maldjian, Laurienti, Kraft, \& Burdette, 2003). Specifically, we used the following ROIs, each separately for the left and right hemisphere: BA 18/19, BA 7, BA 6 , BA 24/32, BA 44/45/47, and BA 10 . Because the voxel with the largest slope was selected, a bias toward higher values was introduced. However, this bias should operate equally for each set size condition and for younger and older adults. Therefore, although the actual values should not be considered as meaningful, the comparisons of slopes for Set

\footnotetext{
${ }^{1}$ A high $Z$ threshold was used in this analysis (which compares the activity during the task with the activity during fixation) to prevent merging all the brain activations into a single, large undifferentiated cluster, given the very large level of activity in this task. More standard and less conservative thresholds were used for all other analyses.

${ }^{2}$ Given the large size of each ROI, and the fact that in such cases function within each ROI may not be unitary, we felt that using the more standard practice of averaging the activity within each ROI would not be appropriate. However, because single voxel analyses may be unreliable, we also repeated the ROI analysis using the average of a "box" encompassing 27 contiguous voxels surrounding the peak as a method for providing a more stable estimate of activity for each subject and condition. The results were virtually identical to those obtained with the single voxel analysis. All of the effects that were significant at a $p<.05$ level with the single voxel analysis were also significant with the 27 -voxel analysis, and those that were not still remained nonsignificant. The 27 -voxel analysis is available as supplementary material.
} 
Sizes 2-4 and 4-6 and the comparisons between younger and older adults are legitimate. This procedure was selected over alternatives (e.g., using a fixed voxel per subject or per group) because these alternative analyses may bias the results against the older group due to increased anatomical variability with age, which must be taken into consideration given the numerous findings of brain matter loss with age (Gordon et al., 2008; Rettmann, Kraut, Prince, \& Resnick, 2006; Resnick et al., 2000; Raz et al., 1997). Further, because the fMRI data were spatially filtered, this procedure is analogous to considering the weighted average of the largest adjacent voxels within each ROI.

The resulting peak-voxel data for each ROI were first tested for a significant overall slope from Set Sizes 2-6, collapsed across groups and set size conditions. Those showing a significant slope were then further analyzed to determine the presence of significant slopes within the younger and the older groups separately for both lower (2-4) and higher (4-6) set sizes. We also compared how the two slopes (2-4 and 4-6) differed within each group (younger and older adults).

\section{RESULTS}

\section{Behavioral Results}

The mean RTs and Fisher-corrected accuracy values are presented in Figure 2, separately for each age group, session, and set size condition. Results from the mixed-design ANOVA for RT revealed a significant main effect of set size, $F(4,156)=55.97, p<.01,{ }^{3}$ and a significant main effect of age, $F(1,39)=7.16, p<.05$, indicating that both younger and older adults were slower with increasing memory load and that older adults, overall, were significantly slower than younger adults. The Set Size $\times$ Age interaction was not significant, $F(4,156)=0.56, n s$, indicating that the increase in RT with increasing memory load was not significantly different for younger and older adults. To keep behavioral analyses in line with those of fMRI data, we also performed two-tailed $t$ tests, directly comparing the slopes for the younger and the older adults for low (2-4) and high (4-6) set sizes. We also compared the slopes for low and high set sizes separately for younger and older adults. These comparisons revealed no significant effects (for details, see Table 2).

The accuracy analysis also showed a main effect of set size, $F(4,156)=11.47, p<.01$, and a main effect of age, $F(1,39)=12.08, p<.01$, indicating that both younger and older adults were less accurate for higher memory loads and that the older adults were less accurate compared with the younger adults, respectively. The Set Size $\times$ Age interaction was marginally significant, $F(4,156)=2.05, p<.10$.

The throughput data are shown in Figure 3. The younger adults' function approached the ideal function, but with a shallower slope. In other words, younger adults were able, on average, to increase throughput up to 4.98 items, occurring at Set Size 6, whereas older adults only showed an increase up to 3.46 items, occurring at Set Size 5 with no additional information throughput for Set Size 6. The separate planned $t$ tests revealed significantly different slopes between groups for low and high set sizes, with the older adults showing a smaller increase for low set sizes and nearly no increase for high set sizes (see details in Table 2). These data indicate that older adults may be unable to maintain, on average, more than four items in WM because additional items beyond Set Size 4 did not significantly increase their throughput measurement. Younger adults, on the other hand, may retain significantly more information.

\footnotetext{
${ }^{3}$ The degrees of freedom in the behavioral analyses are reduced due to one subject's missing the RT value for Set Size 2 because of response box malfunction.
} 


\section{fMRI Results}

Overall mean contrasts for each age group are presented in Figure 4 and show a number of regions being active during the task. Younger adults showed foci of activation in bilateral occipital, left parietal, left premotor, and left medial frontal cortex. Older adults showed bilateral foci of activation in occipital, parietal, premotor, and medial frontal cortex. These results replicate findings reported in other studies, indicating that WM tasks induce the activation of a dorsal fronto-parietal network (Champod \& Petrides, 2007; Cabeza et al., 2004; Cabeza, Dolcos, Graham, \& Nyberg, 2002; Cornette, Dupont, Salmon, \& Orban, 2001; Jonides et al., 1997), which is left lateralized in the younger adults and bilaterally activated in the older adults. The presence of bilateral activity in older adults in a task showing unilateral activity in younger adults is a common observation in brain imaging studies, as summarized by the HAROLD model (Cabeza, 2002). In addition, bilateral activation of occipital areas was found in both younger and older adults. This may reflect the visual nature of the task.

To examine load effects, we conducted linear trend analyses, separately for low (2-4) and high (4-6) set sizes. These analyses revealed a clear differentiation between the two age groups, with younger adults showing no significant (i.e., subthreshold) linear trends for low set sizes but pronounced linear increases in several areas for high set sizes and older adults showing significant effects at low set sizes but no further significant increases at high set sizes (see Figure 5). ${ }^{4}$ For the younger adults, low set sizes were associated with foci of linear increase only in left occipital cortex, whereas high set sizes were associated with linear increases in bilateral parietal and frontal cortex in addition to the left occipital cortex (see Table 3). The older adults showed foci of linear increase in left occipital, bilateral parietal, bilateral premotor, bilateral inferior frontal, and medial frontal cortex at low set sizes but not at high set sizes.

These data show both overrecruitment (at low set sizes) and underrecruitment (at high set sizes) in older adults, as postulated by the CRUNCH model. Interestingly, however, they also show some bilateral recruitment in younger adults at high loads, suggesting that the recruitment of additional areas may be a common mechanism to deal with increasing task difficulty or load rather than a mechanism geared at compensating for loss in neuronal efficiency that is specific to aging.

To further examine these load effects, we focused our statistical analyses on the peak voxels in the ROIs showing large changes as a function of memory load, which included BA 18/19, BA 7, BA 6, BA 24/32, and BA 44/45/47. For each of these regions, the voxel corresponding to the peak response was identified, separately for each subject, set size condition, and hemisphere. Because the interest of this study is to evaluate differences in brain activation as a function of memory load, it is important to first minimize the impact of individual (or group) differences on the overall magnitude of the brain oxygen-level dependent (BOLD) response. Therefore, we scaled the peak values observed for each subject, hemisphere, and memory set size by the amplitude of the largest response observed across set sizes for each individual subject. These relative amplitude values were then used for all following analyses.

The brain-activation-by-memory-load functions for each ROI peak voxel and for the average across ROIs are presented in Figure 6 (panels B and A, respectively). Results of $t$ test analyses are presented in Table 4 . These data indicate that, although an increase in brain activity as a function of memory load was observed for most areas in both hemispheres, the

\footnotetext{
${ }^{4}$ Note that a lower threshold was used for the younger adults to adjust for the smaller number of subjects in that group.
} 
pattern was quite different for younger and older subjects: Whereas the younger adults showed most of the increase between Load 4 and Load 6, the older adults showed most of the increase between Load 2 and Load 4.

Relationship between Relative fMRI Activation, Memory Load, and WM SpanOne of the most important predictions of the CRUNCH model is that age-related differences in brain activity are a reflection of the more limited processing capacity of the older adults. As a consequence, older adults require a greater amount of brain activation to handle relative low loads. This implies that differences between younger and older adults should disappear once the load is computed relative to each individual's WM span rather than in absolute terms. The throughput measure that we have described earlier can provide a tool for quantifying WM span within the context of the Sternberg memory search task. Specifically, we expect that, when WM span is reached, the throughput measure should reach an asymptote - that is, it should not increase with further increases in memory load. The asymptotic (maximum) value can then be considered as an estimate of WM span, which can be assessed individually for each participant in the study. Because we were interested in using this measure to estimate the brain activation levels relative to memory span, we approximated this value to the nearest integer. The resulting estimates of WM span (based on each person's throughput asymptote) correlated significantly with O-SPAN measures ( $r=$ $0.38, p<.01$, one tailed). This indicates that this measure is a valid estimate of WM span. Importantly, it is also a measure obtained during the same task in which the fMRI was recorded. Interestingly, this measure differed significantly between younger (mean $=5.08$ items) and older adults (mean $=3.80$ items), $t(40)=5.06, p<.0001$, although for both groups the estimates were relatively close to the memory span ranges given by Cowan (2001).

Another useful characteristic of this measure is that it allows us to evaluate, for each individual subject, what amount of brain activity is required as a function of the relationship between memory load and WM span. The results of this analysis, averaged across all the ROIs that showed significant increases of BOLD response as a function of relative set size and across all subjects in the study, are presented in Figure 6C.

The data presented in this figure indicate that the BOLD response increased in a sigmoid (rather than linear) fashion. For very low memory loads (relative to WM span), the curve was essentially flat. However, when memory loads were closer to span, the curve rose steeply but flattened once WM span was attained, reaching an asymptote related to the performance (throughput) asymptote. To confirm this visual impression, a set of $t$ tests were performed for consecutive steps of increasing memory load, corrected for multiple comparisons using the Bonferroni procedure. The step between span -1 and span showed a significant increase in BOLD response, $t(41)=2.98, p<.05$ (Bonferroni corrected). None of the other steps reached statistical significance. Such sigmoid function was evident for both younger and older adults, and in both groups of subjects it reached its asymptote when the load was equal to the WM span. No point in the curve showed a significant difference between younger and older adults (all $t \mathrm{~s}<1$ ). Thus, the difference between the fMRI load functions in younger and older subjects (presented in the upper portion of Figure 6A) disappears when individual subjects' WM spans are taken into consideration. This finding is clearly consistent with CRUNCH. In addition, however, it also underscores the fact that a common mechanism may come into play as subjective load increases, regardless of age (i.e., once individual differences in span are taken into account).

To provide further evidence of the relationship between the increase in BOLD activity with load and WM span, we considered the relationship between the throughput measure representing the behavioral span and the load at which the BOLD response reached its 
asymptote. This was estimated as the first memory load condition at which the fMRI activation reached $80 \%$ of its maximum value. This point was established for each subject across regions. The mean value of the fMRI asymptotic point was larger for the younger ( mean load $=4.67$ items) than for the older adults (mean load $=3.80$ items), $t(40)=2.05, p$ $<.05$, paralleling the difference in span size between the two groups. In fact, the difference between the point of asymptote in the fMRI load function and the span size was similar across groups (younger adults $=0.42$ items; older adults $=0.00$ items; $t=1.01, n s$ ). The relationship between these two measures for individual subjects is presented in Figure 7. This figure indicates a significant correlation between these two measures $(r=.44, p<.002$, one tailed).

\section{DISCUSSION}

Taken together, the results of this study provide strong, quantitative support for the utilization of neural networks proposed by CRUNCH (Reuter-Lorenz \& Cappell, 2008). They further indicate that brain activity is nonlinearly related to WM load. Both of these findings have implications: the first for theories of aging and the second for theories of WM.

The results of this study are largely consistent with previous brain imaging data obtained with the Sternberg task (Bunge et al., 2001; D'Esposito et al., 2000; Henson et al., 2000; Rypma \& D'Esposito, 1999). As in previous work, a large number of areas were activated during this task, including occipital, prefrontal, parietal, and medial areas. ${ }^{5}$ When all memory load conditions were combined together and compared with the rest period (Figure 4), preponderantly left hemisphere activations were observed in younger adults, whereas more bilateral activations were observed in older adults. Although this observation is consistent with previous aging data (see HAROLD; Cabeza et al., 2004; Cabeza, 2002; see also Reuter-Lorenz et al., 1999), this group difference in lateralization was not evident when the memory-load conditions were contrasted with each other, as bilateral differences in activation were evident at high loads even for younger adults (Figure 5; for a similar finding, see also Bunge et al., 2001). It is possible that the age-related differences in lateralization regardless of load may not be related to WM function per se (which should vary with memory load), but to other aspects of the task, such as perceptual and motor function, which may be common to all memory load conditions.

The main purpose of this study was to quantitatively investigate predictions made by CRUNCH (Reuter-Lorenz \& Cappell, 2008). CRUNCH is centered on the idea that differences in overrecruitment and underrecruitment of brain areas commonly observed between younger and older adults may reflect age-related differences in processing capacity (or ability). Older adults, for reasons that are yet to be completely understood, require more resources than younger adults for processing equivalent amounts of information. For this reason, they require additional recruitment of brain activity at lower task loads than younger adults.

In our study, we manipulated task load in a parametric fashion, using memory set sizes varying between 2 and 6 . The behavioral data indicated that older adults, on average, had significantly more problems with the task than younger adults. This was particularly true at high (>4) memory set sizes. In fact, in these conditions, their accuracy declined, and the

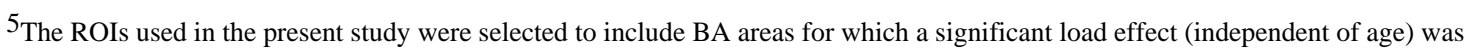
observed. No BA area in dorsolateral prefrontal cortex (DLPFC) made this cut. However, we did observe activation in DLPFC (e.g., in BA 10) during the task, but this activity did not significantly differ as a function of load. It is possible that the apparent lack of load effects in DLPFC is due to the relatively short duration of the maintenance period in the Sternberg task used in this study when compared with that of previous fMRI studies using the same paradigm (e.g., Rypma \& D'Esposito, 1999) or to the continuous maintenance required by the $n$-back task used by Mattay et al. (2006).
} 
"throughput" analysis revealed that they reached a ceiling in their capacity to transmit information (i.e., to correctly identify, above chance, different targets in the presence of increasing information) at about four items. This number was significantly smaller than that for younger adults $(>5)$. In other words, the two age groups differed by more than one full item in terms of their memory span.

This asymptotic performance level in the older adults was associated with a clear asymptotic level in brain activation, as measured with fMRI. The fMRI data indicated that a number of regions, namely, occipital cortex, pre-frontal regions, dorsal parietal cortex, and cingulate cortex, showed significant bilateral increases of activity with set size, presumably reflecting the greater load that a large memory set imposes on the information processing system. In all these areas, the activation-by-set-size functions suggested, for the older adults, a large increase between Set Size 2 and Set Size 4 and a small-to-negligible further increase at larger set sizes (see Figure 6). This pattern contrasts quite obviously with the data obtained in the younger group. Although set size effects were observed in similar areas in younger and older adults, the younger group showed smaller growth in brain activation as a function of set size until at least Set Size 4. A pronounced increase in brain activity in the younger group was observed at higher set sizes.

If the fMRI data were observed in isolation, various hypotheses could be entertained about the significance of these effects. For instance, it could be argued that the neurovascular system is limited in its capacity to provide additional oxygenated blood (and therefore flush out deoxyhemoglobin leading to the BOLD signal) and that this limit is reached at lower loads in older than younger adults. This would explain the earlier asymptote in the older (occurring at Set Size 4) than in the younger subjects (occurring at Set Size 6 or beyond). Although a possible role of an impaired neurovascular system on brain function cannot be ruled out by the present study, the behavioral data clearly indicate that the older adults reach a performance asymptote at about Set Size 4-a value that represents a real limit in processing capacity rather than a mere artifact of the measuring system.

Thus, the data indicate that, whatever the reason, older adults reach an asymptote in both behavior and brain activation at lower levels than younger adults. The individualized span analysis provides an even stronger quantitative support for CRUNCH. It shows that the differences in the brain-activation-by-memory-load function between younger and older adults can be entirely accounted for by differences in span across individuals, regardless of their age. When these differences are taken into account, the curves for younger and older adults are virtually identical. Thus, no special mechanism is required to account for the different pattern of brain activity in older adults with respect to younger adults, as this difference is explained by relative task difficulty. Although some extant data (e.g., Stern et al., 2005) are consistent with the premises of CRUNCH (explicated in Figure 2 of ReuterLorenz \& Cappell, 2008), the current data demonstrate for the first time that relative task difficulty alone is sufficient to account for all of the age-related brain activation differences, and no other mechanism is required. This is made possible by the use of an individualized measure of task-related ability, the throughput measure.

The activation-by-task-load functions are also useful to highlight another important observation. Namely, they are clearly nonlinear, showing two asymptotes: a floor at low set sizes and a ceiling at high set sizes, separated by a region of rapid growth when memory load is approaching span. The presence of a ceiling can be easily explained: At some level, the brain is not capable of producing further activation (at least as measured with fMRI; see also Reuter-Lorenz \& Cappell, 2008). This is associated with a ceiling in performance (when memory span is reached). Although causality cannot typically be inferred with brain imaging data alone, it is very tempting to hypothesize that a common mechanism leads to 
both activation and performance asymptotes. This common mechanism may be a limited capacity of the WM system (a "cognitive" explanation) or a limited capacity of the neurovascular supply to the brain (an "energetic" explanation). The current data are insufficient to tease apart these hypotheses, and in fact there may be no need to do that, as one could argue that the neurovascular system is built to satisfy functional requirements, and therefore it can be expected that the two limits should coincide. Further, the current study cannot be used to determine which specific process of the many involved during the Sternberg task is the one responsible for the limitations in performance and brain activation. The blocked design and fast pace of the study do not allow us to use the relatively slow hemodynamic data provided by fMRI to determine whether the effects are due to processes occurring during encoding, maintenance, or retrieval of information from WM. Other studies based on event-related fMRI designs, often with longer and variable delays (e.g., Grady, Yu, \& Alain, 2008; Rypma et al., 2005; Rypma \& D'Esposito, 2000), or other brain imaging methods with higher temporal resolution such as ERPs or event-related optical signal (Fabiani \& Gratton, 2005; Gratton \& Fabiani, 2001) can be more useful for this purpose.

The presence of a floor effect in the brain imaging data is not predicted by or related to CRUNCH and requires some further consideration. There are three possible explanations for this phenomenon. First, the floor effect may be an artifact due to the insensitivity of the hemodynamic measures to lower levels of brain activation, especially when thresholding is used for statistical analyses. This possibility is difficult to rule out completely, although the effect sizes present in this study are in line with many other published reports. A more interesting explanation for the floor effect is that it is related to the presence of a real floor in brain activation. There are two possible interpretations for this. The first, which is embedded in the model proposed by Cowan (2001), is that WM can itself be partitioned into an easily accessible core of highly activated nodes (whose use requires little effort) and a "halo" of less highly activated nodes (whose use requires more substantial effort). In the present case, when the set size is small, only the core of WM needs to be accessed and very little effort (and brain activity) is required. However, when the set size is large, the halo comes into play, with the consequence that a much greater effort (and brain activity) is involved.

The second interpretation is that the brain activation observed in this task is, to a great extent, related to the necessity of maintaining independent chunks of information active in WM. In other words, the difficulty is not in maintaining the information in an active form but in maintaining the different pieces of information as distinct and eliminating cross talk. In this case, the amount of activity should be related to the number of negative cross-links that need to be established between the different chunks, which should grow in a combinatory (or exponential) fashion with the number of active chunks. According to this interpretation, brain activation should grow exponentially until a ceiling is reached (due to lack of sufficient resources for keeping so many different representations distinct from each other). Hence, the brain-activation-by-set-size function should be a sigmoid, with the largest growth occurring around span. A depiction of this theoretical view is presented in Figure 8.

Within this framework, the main difference between younger and older adults is that the sigmoid function relating memory load to brain activity and performance is shifted to the left in older adults (which is consistent with the model proposed by Rypma, Eldreth, \& Rebbechi, 2007). For either of the "cognitive" accounts described above, we would need to determine why older adults have, on average, a smaller span than younger adults, although their respective functions are very similar when scaled by the individual spans. With respect to the first hypothesis, this would suggest that the difference between younger and older adults is in the size of the core area, which could reflect the ability to maintain focus on particular memory nodes that carry task-relevant information. This may in turn be seen as an 
attention control function, as in fact proposed by both Engle and Kane (2004) and Cowan (2001). In this case, older adults may have a smaller WM span because they have poorer control of attention - in other words, the WM core may be less stable regardless of its size. There is in fact substantial evidence that older adults may have problems controlling attention and be more distractible than younger adults (Hasher et al., 2008; Hasher \& Zacks, 1988). This in itself may reflect difficulties in suppressing the processing of irrelevant information and be related to a general deficit in inhibitory processes (e.g., Fabiani et al., 1998, 2006; Gazzaley, Cooney, Rissman, \& D’Esposito, 2005; Fabiani \& Friedman, 1995).

Interestingly, inhibitory processes may also be very important for maintaining separate memory representations - the crucial element in our second hypothesis. The inhibitory deficits commonly observed in older adults may then account for the difficulty in maintaining separate memory representations: The increased brain activity observed at lower loads in older adults may then reflect the requirement to overcome the deficit in reciprocal inhibition between different memory representations. In fact, the two hypotheses described above may be two sides of the same coin: In both cases, deficits in inhibitory processes may lead to an inability to maintain attentional focus and to keep different memory representations active and distinct and in turn limit the effective size of WM span.

In conclusion, the results of this study provide strong quantitative support for CRUNCH and in particular for the relative utilization of neural networks depending on performance. They are less conclusive, however, regarding the idea of compensation also embedded in CRUNCH. The difference in brain activation as a function of load that exists between younger and older adults can be entirely accounted for by the difference in their WM capacity. In other words, the data indicate that, given equal objective memory loads, individuals with lower memory abilities are deploying more brain activation than those with higher memory abilities, regardless of age. To the extent that "compensation" lis intended as the amount of effort needed to reach a given level of performance, the data are consistent with the predictions of the compensation hypothesis. However, because of their correlational nature, the data do not provide conclusive information about the causal direction of the relationship between brain activity and behavior: We cannot say whether the increased brain activity is used to improve performance or whether the lack of some general ability causes both the increased brain activity and the decrement in performance. The data also show that the brain-activation-by-memory-load functions are nonlinear, displaying both a ceiling and a floor effect. The ceiling effect is associated with a limit in WM span. The floor effect may be a reflection of the large difference in the mental effort required to maintain an increasing number of items active and/or distinct within WM. This mental effort is likely to be largely related to inhibitory processes, which may be impaired in older adults.

\section{Acknowledgments}

This work was supported in part by NIA grant \#AG21887 to Monica Fabiani. We wish to thank Kirk Erikson and Paige Scalf for advice regarding the fMRI analysis and Nelson Cowan and Kathy Low for helpful comments on an earlier version of this manuscript.

\section{References}

Baddeley, AD. Working memory. New York: Oxford University Press; 1986.

Baddeley, AD.; Hitch, G. Working memory. In: Bower, G., editor. Recent advances in learning and motivation. Vol. 8. New York: Academic Press; 1974. p. 47-90.

Beck, AT.; Steer, RA. Manual for the Beck Depression Inventory. San Antonio, TX: Psychological Corporation; 1996.

Beckmann CF, Jenkinson M, Smith SM. General multilevel linear modeling for group analysis in fMRI. Neuroimage. 2003; 20:1052-1063. [PubMed: 14568475] 
Bopp KL, Verhaeghen P. Aging and verbal memory span: A meta-analysis. Journals of Gerontology, Series B, Psychological Sciences and Social Sciences. 2005; 60:223-233.

Bunge SA, Ochsner KN, Desmond JE, Glover GH, Gabrieli JDE. Prefrontal regions involved in keeping information in and out of mind. Brain. 2001; 124:2074-2086. [PubMed: 11571223]

Cabeza R. Hemispheric asymmetry reduction in older adults: The HAROLD model. Psychology and Aging. 2002; 17:85-100. [PubMed: 11931290]

Cabeza R, Daselaar SM, Dolcos F, Prince SE, Budde M, Nyberg L. Task-independent and taskspecific age effects on brain activity during working memory, visual attention and episodic retrieval. Cerebral Cortex. 2004; 14:364-375. [PubMed: 15028641]

Cabeza R, Dolcos F, Graham R, Nyberg L. Similarities and differences in the neural correlates of episodic memory retrieval and working memory. Neuroimage. 2002; 16:317-330. [PubMed: 12030819]

Champod AS, Petrides M. Dissociable roles of the posterior parietal and the prefrontal cortex in manipulation and monitoring processes. Proceedings of the National Academy of Sciences, USA. 2007; 104:14837-14842.

Cornette L, Dupont P, Salmon E, Orban GA. The neural substrate of orientation working memory. Journal of Cognitive Neuroscience. 2001; 13:813-828. [PubMed: 11564325]

Cowan N. The magical number 4 in short-term memory: A reconsideration of mental storage capacity. Behavioral and Brain Sciences. 2001; 24:87-114. [PubMed: 11515286]

Cowan N, Elliot EM, Scott-Saults J, Moray CC, Mattox S, Hismjatullina A, et al. On the capacity of attention: Its estimation and its role in working memory and cognitive aptitudes. Cognitive Psychology. 2005; 51:42-100. [PubMed: 16039935]

Craik, F. Short-term memory and the aging process. In: Talland, G., editor. Human aging and behavior. New York: Academic Press; 1968. p. 131-168.

Craik, FIM.; Byrd, M. Aging and cognitive deficits: The role of attentional resources. In: Craik, FIM.; Trehub, S., editors. Aging and cognitive processes. New York: Plenum Press; 1982. p. 191-211.

D'Esposito M, Postle BR, Rypma B. Prefrontal cortical contributions to working memory: Evidence from event-related fMRI studies. Experimental Brain Research. 2000; 133:3-11.

Engle, RW.; Kane, MJ. Executive attention, working memory capacity, and a two-factor theory of cognitive control. In: Ross, B., editor. The psychology of learning and motivation. Vol. 44. New York: Elsevier; 2004. p. 145-199.

Fabiani M, Friedman D. Changes in brain activity patterns in aging: The novelty oddball. Psychophysiology. 1995; 32:579-594. [PubMed: 8524992]

Fabiani M, Friedman D, Cheng JC. Individual differences in P3 scalp distribution in older adults, and their relationship to frontal lobe function. Psychophysiology. 1998; 35:698-708. [PubMed: 9844431]

Fabiani, M.; Gratton, G. Electrophysiological and optical measures of cognitive aging. In: Cabeza, R.; Nyberg, L.; Park, D., editors. Cognitive neuroscience of aging: Linking cognitive and cerebral aging. New York: Oxford University Press; 2005. p. 85-106.

Fabiani M, Low KA, Wee E, Sable JJ, Gratton G. Reduced suppression or labile memory? Mechanisms of inefficient filtering of irrelevant information in older adults. Journal of Cognitive Neuroscience. 2006; 18:637-650. [PubMed: 16768366]

Gazzaley A, Cooney JW, Rissman J, D’Esposito M. Top-down suppression deficit underlies working memory impairment in normal aging. Nature Neuroscience. 2005; 8:1298.

Gordon B, Rykhlevskaia E, Brumback CR, Lee Y, Elavsky S, Konopack JF, et al. Anatomical correlates of aging, cardiopulmonary fitness level, and education. Psychophysiology. 2008; 45:825-838. [PubMed: 18627534]

Grady CL, Maisog JM, Horwitz B, Ungerleider LG, Mentis MJ, Salerno JA, et al. Age-related changes in cortical blood flow activation during visual processing of faces and location. Journal of Neuroscience. 1994; 14:1450-1462. [PubMed: 8126548]

Grady CL, Yu H, Alain C. Age-related differences in brain activity underlying working memory for spatial and nonspatial auditory information. Cerebral Cortex. 2008; 18:189-199. [PubMed: 17494060] 
Gratton G, Fabiani M. Shedding light on brain function: The event-related optical signal. Trends in Cognitive Science. 2001; 5:357-363.

Hasher, L.; Lustig, C.; Zacks, R. Inhibitory mechanisms and the control of attention. In: Conway, A.; Jarrold, C.; Kane, M.; Miyake, A.; Towse, J., editors. Variation in working memory. New York: Oxford University Press; 2008. p. 227-249.

Hasher L, Zacks RT. Working memory, comprehension, and aging: A review and a new view. Psychology of Learning and Motivation. 1988; 22:193-225.

Henson RNA, Burgess N, Frith CD. Recoding, storage, rehearsal and grouping in verbal short-term memory: An fMRI study. Neuropsychologia. 2000; 38:426-440. [PubMed: 10683393]

Jonides J, Schumacher EH, Smith EE, Lauber EJ, Awh E, Minoshima S, et al. Verbal working memory load affects regional brain activation as measured by PET. Journal of Cognitive Neuroscience. 1997; 9:462-475.

Kane MJ, Bleckley MK, Conway AR, Engle RW. A controlled-attention view of working-memory capacity. Journal of Experimental Psychology: General. 2001; 44:169-183. [PubMed: 11409097]

Kane MJ, Engle RW. Working-memory capacity, proactive interference, and divided attention: Limits on long-term memory retrieval. Journal of Experimental Psychology: Learning, Memory, and Cognition. 2000; 26:336-358.

La Pointe LB, Engle RW. Simple and complex word spans as measures of working memory capacity. Journal of Experimental Psychology: Learning, Memory, and Cognition. 1990; 16:1118-1133.

Lindenberger U, Baltes PB. Intellectual functioning in old and very old age: Cross-sectional results from the Berlin Aging Study. Psychology and Aging. 1997; 12:410-432. [PubMed: 9308090]

Maldjian JA, Laurienti PJ, Kraft RA, Burdette JH. An automated method for neuroanatomic and cytoarchitectonic atlas-based interrogation of fMRI data sets. Neuroimage. 2003 ; 19:1233-1239. [PubMed: 12880848]

Mattay VS, Fera F, Tessitore A, Hariri AR, Berman KF, Das S, et al. Neurophysiological correlates of age-related changes in working memory capacity. Neuroscience Letters. 2006; 392:32-37. [PubMed: 16213083]

Mayeux R, Stern Y, Rosen J, Leventhal J. Depression, intellectual impairment, and Parkinson disease. Neurology. 1981; 31:645-650. [PubMed: 7195481]

McIntosh AR, Sekuler AB, Penpeci C, Rajah MN, Grady CL, Sekuler R, et al. Recruitment of unique neural systems to support visual memory in normal aging. Current Biology. 1999; 9:1275-1278. [PubMed: 10556091]

Miller GA. The magical number seven, plus or minus two: Some limits on our capacity for processing information. Psychological Review. 1956; 63:81-97. [PubMed: 13310704]

Oldfield RC. The assessment and analysis of handedness: The Edinburgh inventory. Neuropsychologia. 1971; 9:97-113. [PubMed: 5146491]

Park DC, Lautenschlager G, Hedden T, Davidson NS, Smith AD, Smith PK. Models of visuospatial and verbal memory across the adult life span. Psychology and Aging. 2002; 17:299-320. [PubMed: 12061414]

Park DC, Welsh RC, Marshuetz C, Gutchess AH, Mikels J, Polk TA, et al. Working memory for complex scenes: Age differences in frontal and hippocampal activations. Journal of Cognitive Neuroscience. 2003; 15:1122-1134. [PubMed: 14709231]

Payer D, Marshuetz C, Sutton B, Hebrank A, Welsh RC, Park DC. Decreased neural specialization in old adults on a working memory task. NeuroReport. 2006; 17:487-491. [PubMed: 16543812]

Raz N, Gunning FM, Head D, Dupuis JH, McQuain J, Briggs SD, et al. Selective aging of the human cerebral cortex observed in vivo: Differential vulnerability of the prefrontal gray matter. Cerebral Cortex. 1997; 7:268-282. [PubMed: 9143446]

Resnick SM, Goldszal AF, Davatzikos C, Golski S, Kraut MA, Metter EJ, et al. One-year age changes in MRI brain volumes in older adults. Cerebral Cortex. 2000; 10:464-472. [PubMed: 10847596]

Rettmann ME, Kraut MA, Prince JL, Resnick SM. Cross-sectional and longitudinal analyses of anatomical sulcal changes associated with aging. Cerebral Cortex. 2006; 16:1584-1594. [PubMed: 16400155]

Reuter-Lorenz PA, Cappell KA. Neurocognitive aging and the compensation hypothesis. Current Directions in Psychological Science. 2008; 17:177-182. 
Reuter-Lorenz PA, Lustig C. Brain aging: Reorganizing discoveries about the aging mind. Current Opinion in Neurobiology. 2005; 15:245-251. [PubMed: 15831410]

Reuter-Lorenz PA, Marshuetz C, Jonides J, Smith EE. Neurocognitive aging of storage and executive processes. European Journal of Cognitive Psychology. 2001; 13:257-258.

Reuter-Lorenz PA, Stanczak L, Miller AC. Neural recruitment and aging: Two hemispheres are better than one, especially as you age. Psychological Science. 1999; 10:494-500.

Riecker A, Gröschel K, Ackermann H, Steinbrink C, Witte O, Kastrup A. Functional significance of age-related differences in motor activation patterns. Neuroimage. 2006; 32:1345-1354. [PubMed: 16798017]

Rypma B, Berger JS, D'Esposito M. The influence of working-memory demand and subject performance on prefrontal cortical activity. Journal of Cognitive Neuroscience. 2002; 14:721-731. [PubMed: 12167257]

Rypma B, Berger JS, Genova HM, Rebbechi D, D'Esposito M. Dissociating age-related changes in cognitive strategy and neural efficiency using event-related fMRI. Cortex. 2005; 41:582-594. [PubMed: 16042034]

Rypma B, D'Esposito M. The roles of prefrontal brain regions in components of working memory: Effects of memory load and individual differences. Proceedings of the National Academy of Sciences, USA. 1999; 96:6558-6563.

Rypma B, D'Esposito M. Isolating the neural mechanisms of age-related changes in human working memory. Nature Neuroscience. 2000; 3:509-515.

Rypma B, D'Esposito M. Age-related changes in brain-behavior relationships: Evidence from eventrelated functional MRI studies. European Journal of Cognitive Psychology. 2001; 13:235-256.

Rypma B, Eldreth DA, Rebbechi D. Age-related differences in activation-performance relations in delayed-response task: A multiple component analysis. Cortex. 2007; 43:65-76. [PubMed: 17334208]

Salthouse TA. The processing-speed theory of adult age differences in cognition. Psychological Review. 1996; 103:403-428. [PubMed: 8759042]

Spearman, CE. The abilities of man. New York: MacMillan ; 1927.

Stern Y, Habeck C, Moeller J, Scarmeas N, Anderson KE, Hilton HJ, et al. Brain networks associated with cognitive reserve in healthy young and old adults. Cerebral Cortex. 2005; 15:394-402. [PubMed: 15749983]

Sternberg S. High-speed scanning in human memory. Science. 1966; 153:652-654. [PubMed: 5939936]

Todd JJ, Marois R. Posterior parietal cortex activity predicts individual differences in visual short-term memory capacity. Cognitive, Affective, and Behavioral Neuroscience. 2005; 5:144-155.

Veltman DJ, Rombouts SARB, Dolan RJ. Maintenance versus manipulation in verbal working memory revisited: An fMRI study. Neuroimage. 2003; 18:247-256. [PubMed: 12595179]

Verhaeghen P, Salthouse TA. Meta-analyses of age-cognition relations in adulthood: Estimates of linear and nonlinear age effects and structural models. Psychological Bulletin. 1997; 122:231-249. [PubMed: 9354147]

Wechsler, D. Wechsler Adult Intelligence Scale-Revised. New York: Psychological Corporation; 1981.

Woolrich MW, Behrens TE, Beckmann CF, Jenkinson M, Smith SM. Multilevel linear modelling for fMRI group analysis using Bayesian inference. Neuroimage. 2004; 21:1732-1747. [PubMed: 15050594]

Worsley KJ, Evans AC, Marrett S, Neelin P. A three-dimensional statistical analysis for CBF activation studies in human brain. Journal of Cerebral Blood Flow and Metabolism. 1992; 12:900918. [PubMed: 1400644]

Zarahn E, Rakitin B, Abela D, Flynn J, Stern Y. Age-related changes in brain activation during a delayed item recognition task. Neurobiology of Aging. 2007; 28:784-798. [PubMed: 16621168] 


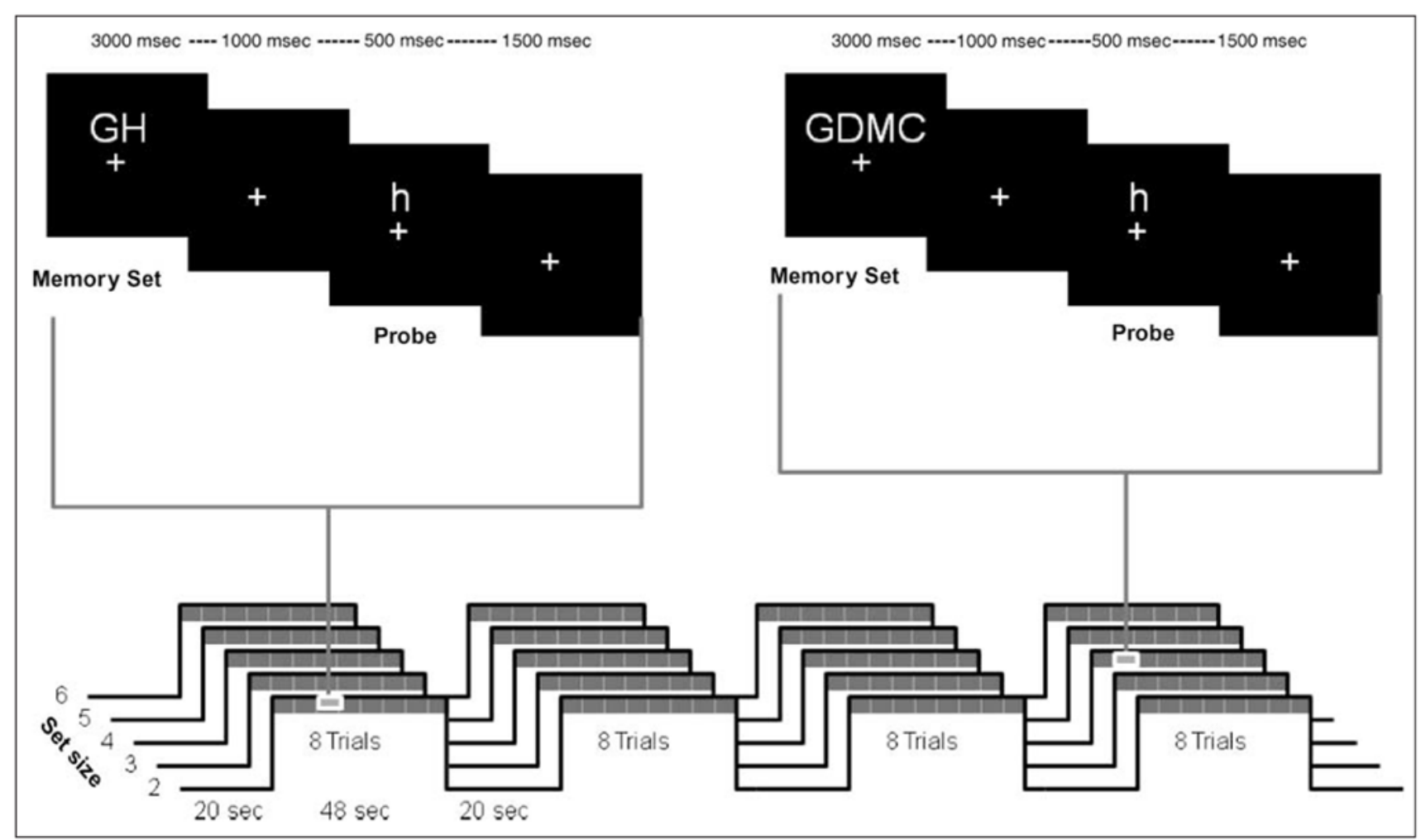

Figure 1.

Procedures and time line. Top: example trials for Set Sizes 2 and 4. Bottom: order of trials for one subject. 


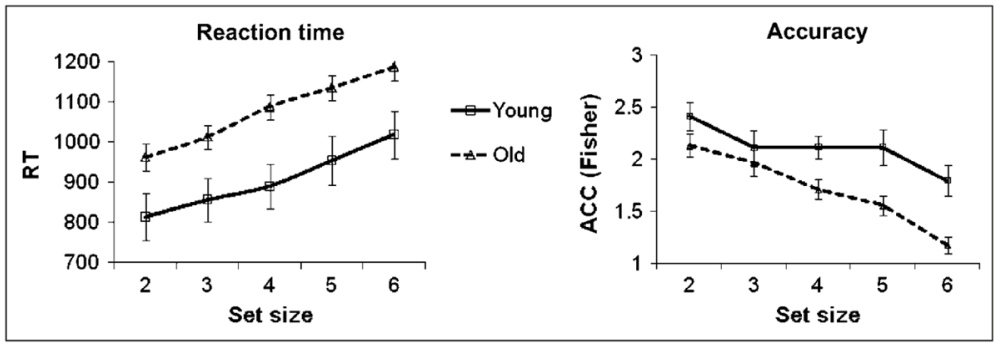

Figure 2.

Mean RT (left) and Fisher-transformed accuracy (right) across set sizes for younger and older adults with estimated standard errors of the means. 


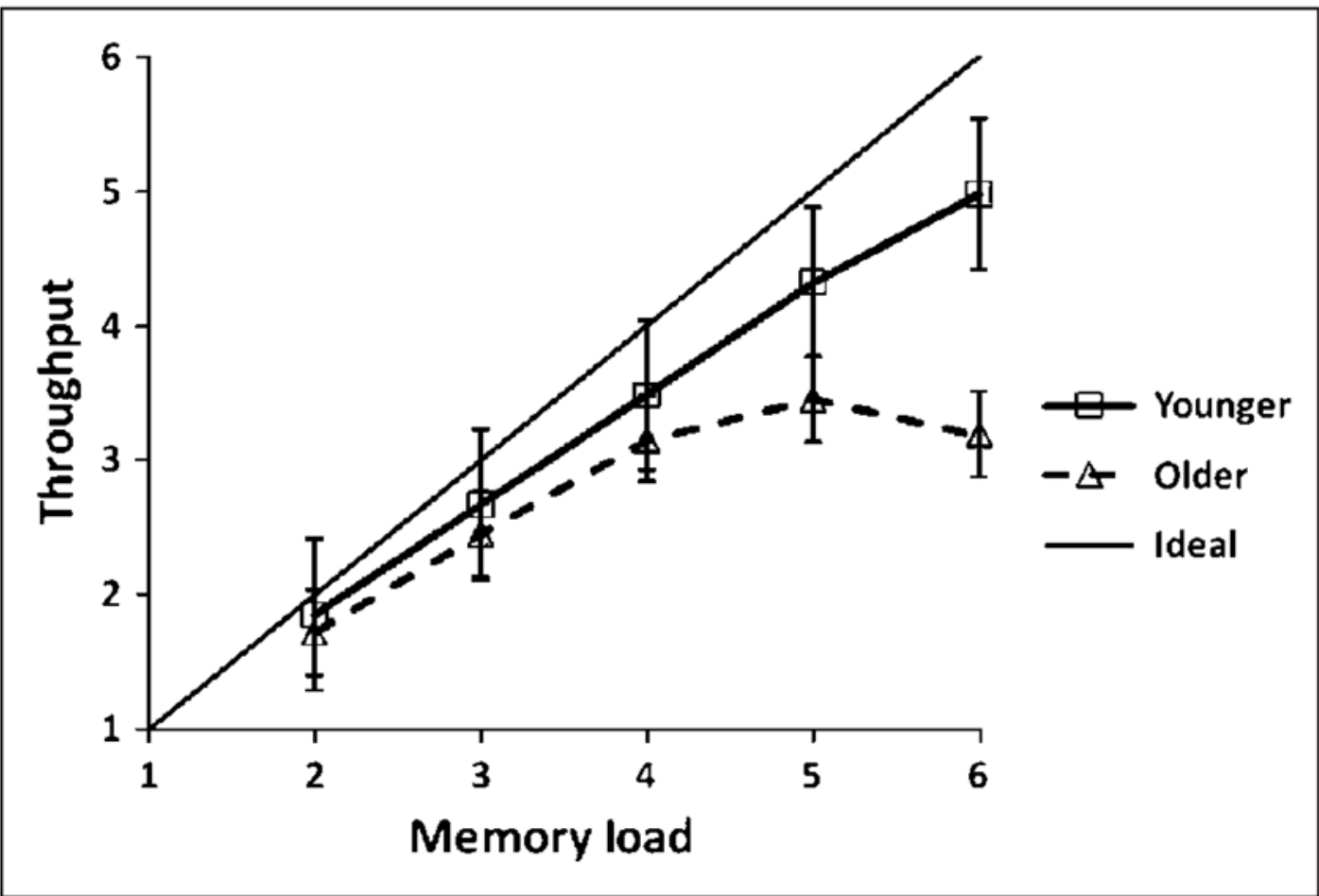

Figure 3.

WM capacity measured by throughput as a function of set size, separately for younger and older adults, with estimated standard errors of the means. The ideal function (accuracy $=1$ ) is provided for reference purposes. 


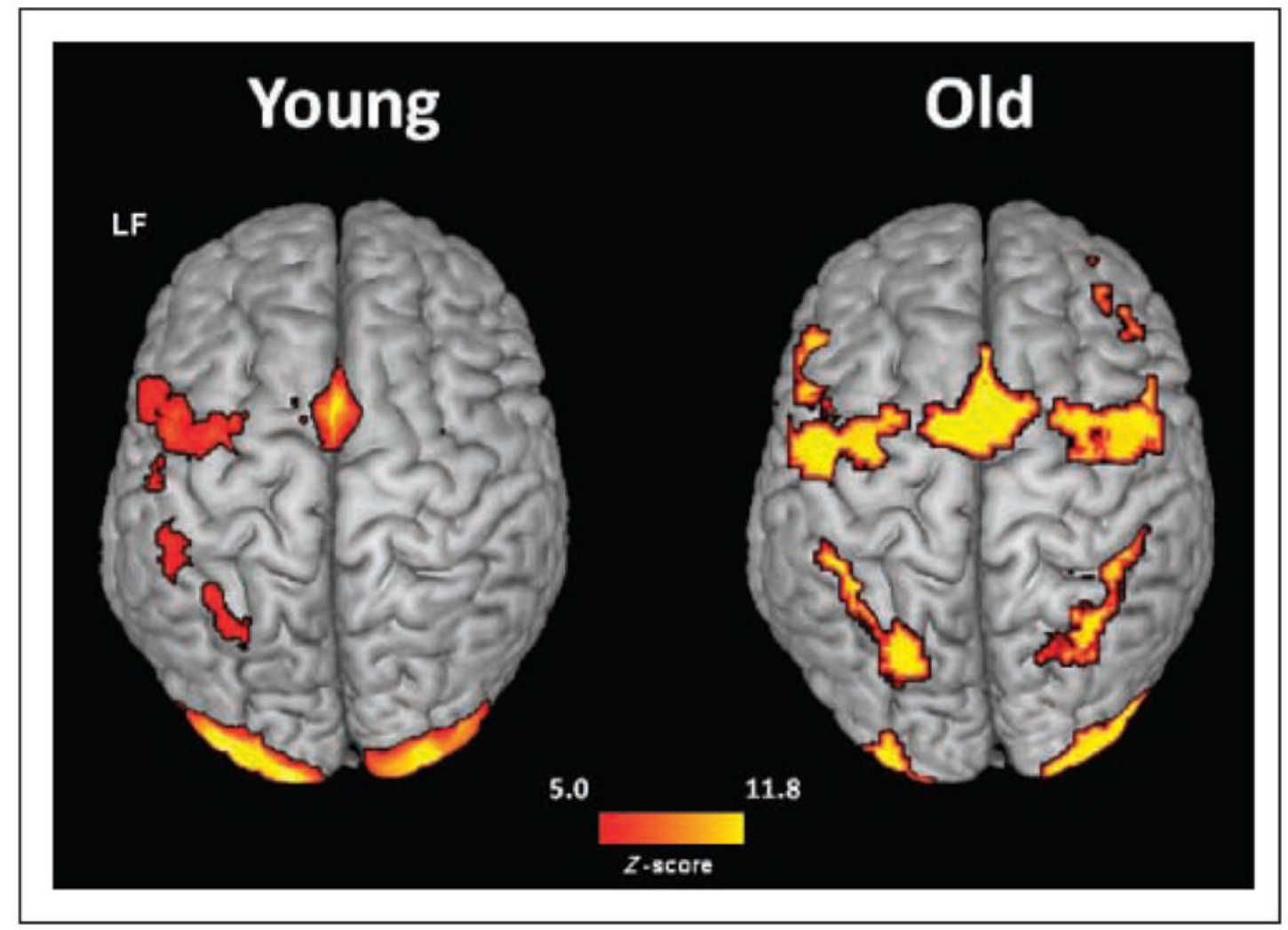

Figure 4.

Statistical brain maps (axial surface projection) of the task minus rest contrast for younger and older adults, collapsed across set sizes. LF $=$ left front. 


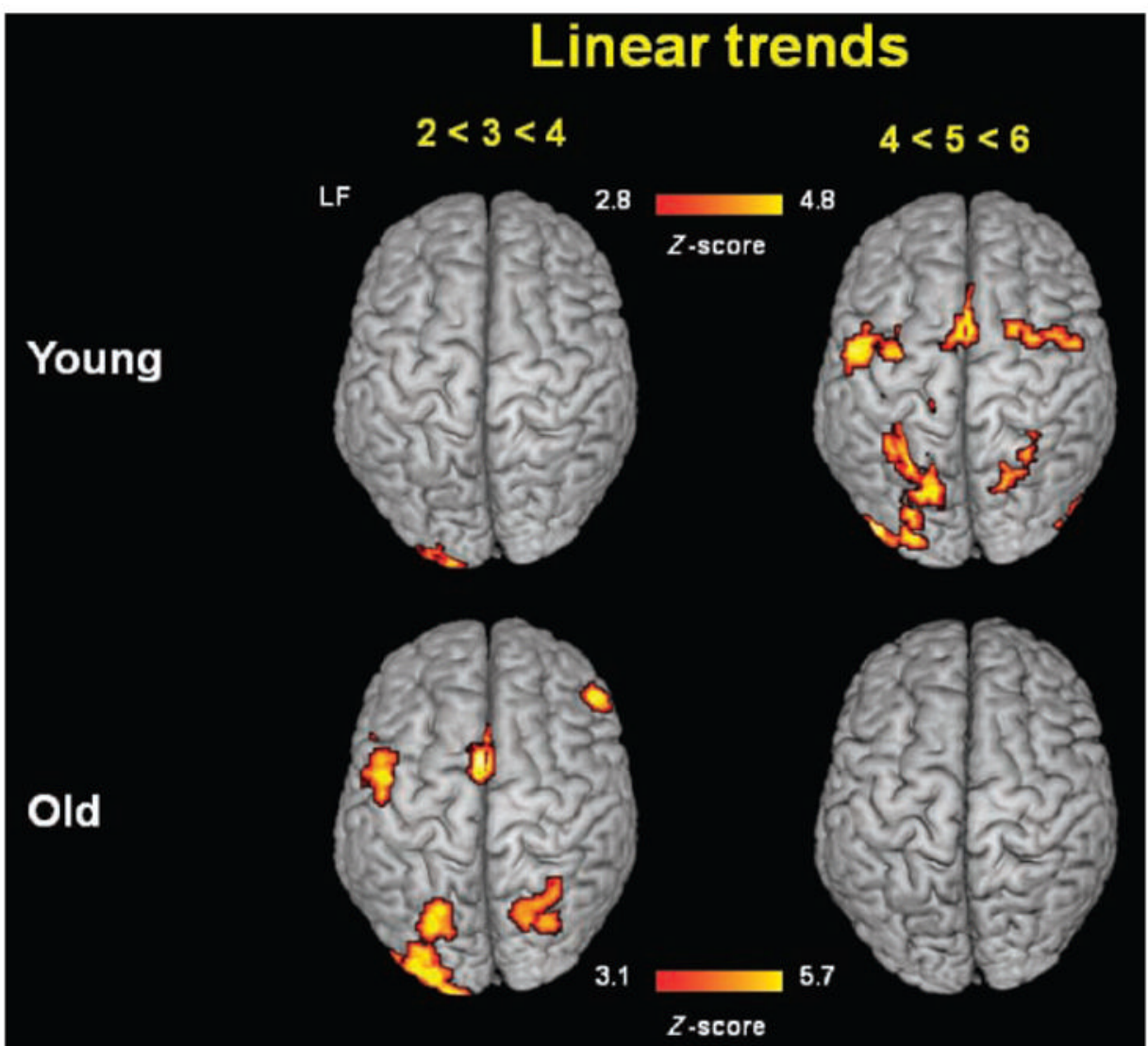

Figure 5.

Statistical brain maps (axial surface projection) of linear trend analyses for Set Sizes 2-4 and 4-6 for younger and older adults. $\mathrm{LF}=$ left frontal. 


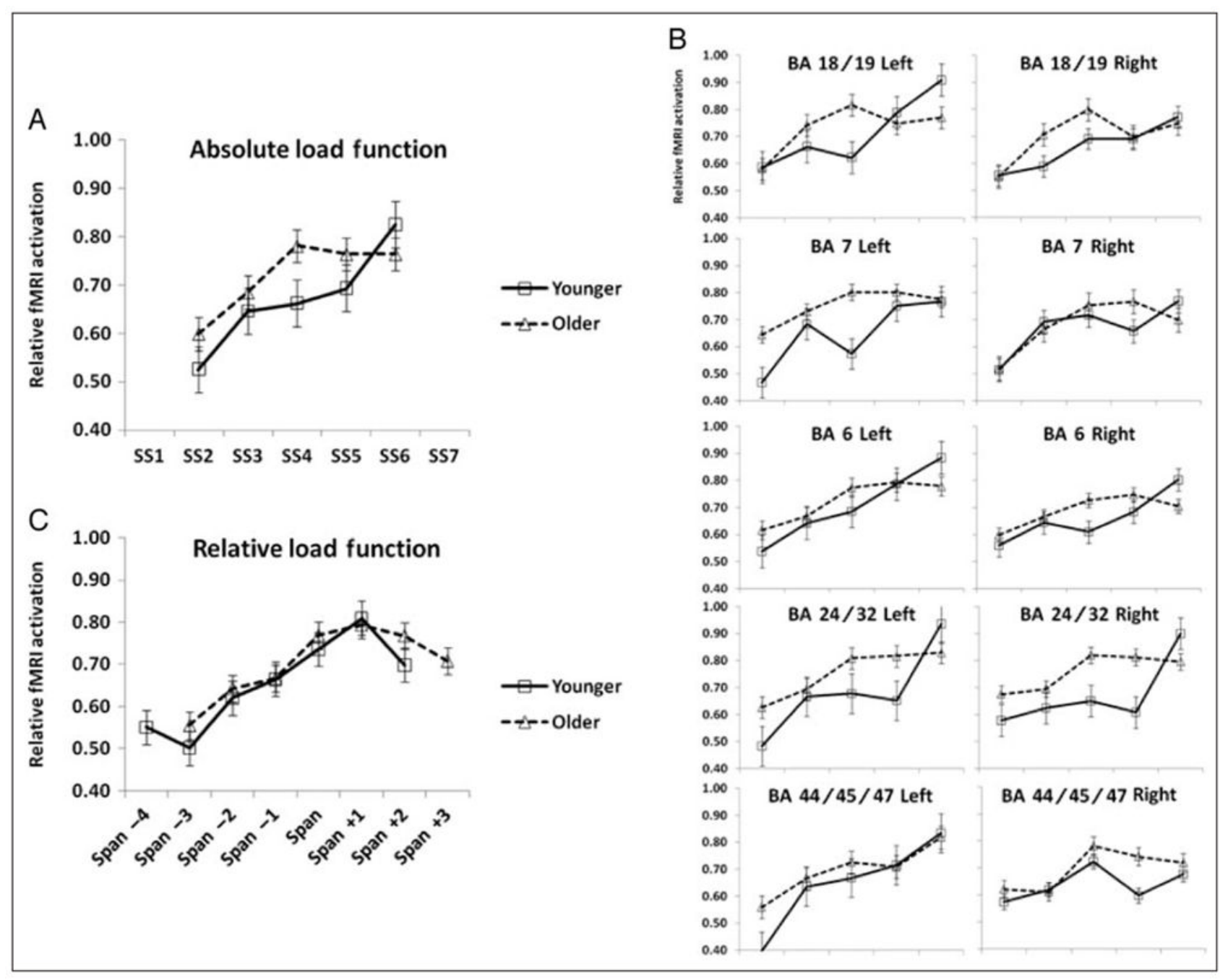

Figure 6.

(A) Relative signal change of BOLD response as a function of set size, averaged across ROIs (peak values) in younger and older adults. (B) Relative signal change of BOLD response as a function of set size, separately for each ROI (peak values). (C) Relative signal change of BOLD response as a function of set size, averaged across ROIs (peak values), when WM load is adjusted as a function of each participant's span. 


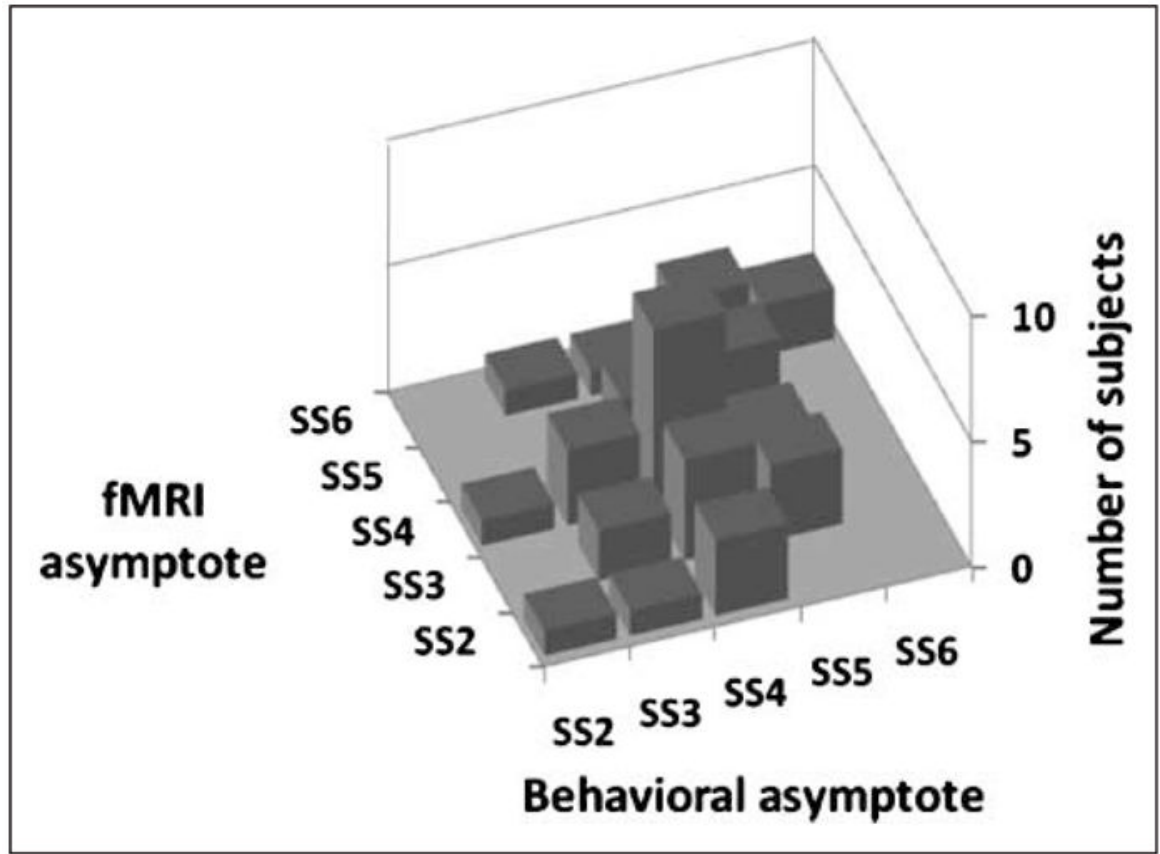

Figure 7.

Three-dimensional scatter plot illustrating the relationship between the point of asymptote in the fMRI activation function and the WM span (maximum value in the throughput function). $z$-Axis $=$ number of subjects; $x$-axis $=$ behavioral asymptote; $y$-axis $=$ fMRI asymptote. 


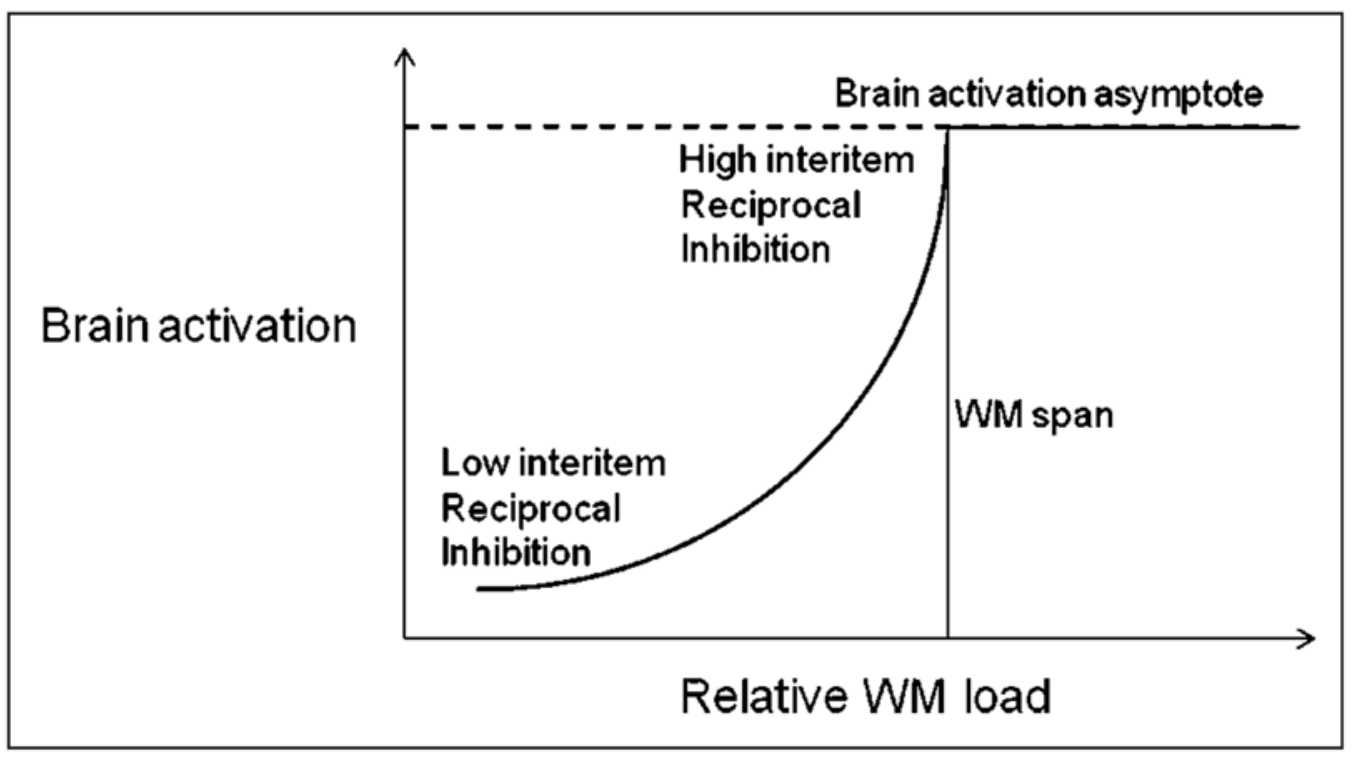

Figure 8.

Schematic depiction of the relationship between subjective WM load and related brain activation. The average function of older adults is expected to be shifted toward the left, that of younger adults toward the right. 


\section{Table 1}

Mean (with Estimated Standard Error in Parentheses) Demographic Characteristics for Younger and Older Adults

\begin{tabular}{lccc}
\hline Measure & Young $(\mathbf{n = 1 2})$ & Old $(\mathbf{n}=\mathbf{3 0})$ & t Test \\
\hline Age (years) & $23.8(0.7)$ & $70.9(0.8)$ & \\
Education (years) & $16.4(0.7)$ & $16.1(0.6)$ & 0.23 \\
Modified Mini-Mental Status examination & $56.7(0.2)$ & $55.5(0.3)$ & $2.37^{* *}$ \\
Vocabulary subscore of Wechsler Adult Intelligence Scale-Revised & $13.0(1.0)$ & $13.3(0.4)$ & 0.36 \\
O-SPAN & $25.0(4.1)$ & $13.9(1.5)$ & $3.33^{* * *}$ \\
\hline
\end{tabular}

$t$ tests between groups (two tailed): $d f=40$.

**

*** $p<.05$.

$* * * * 01$. 


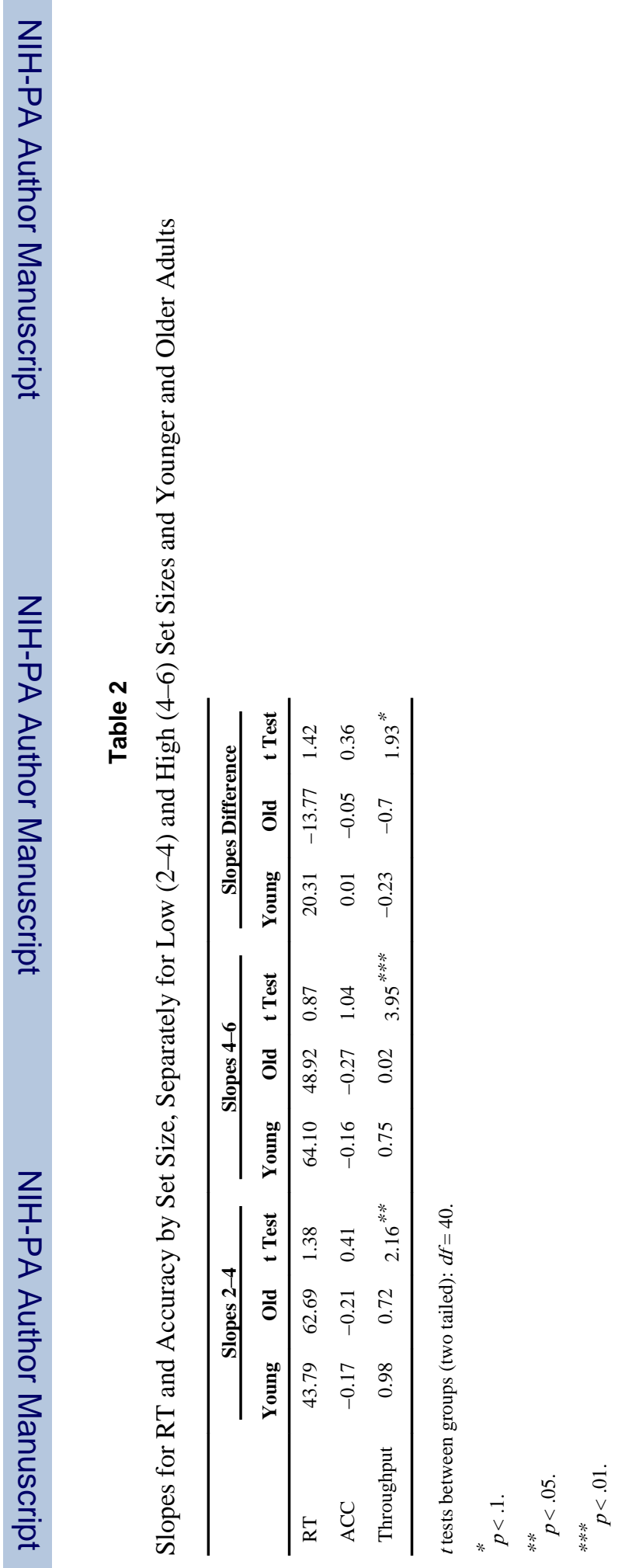




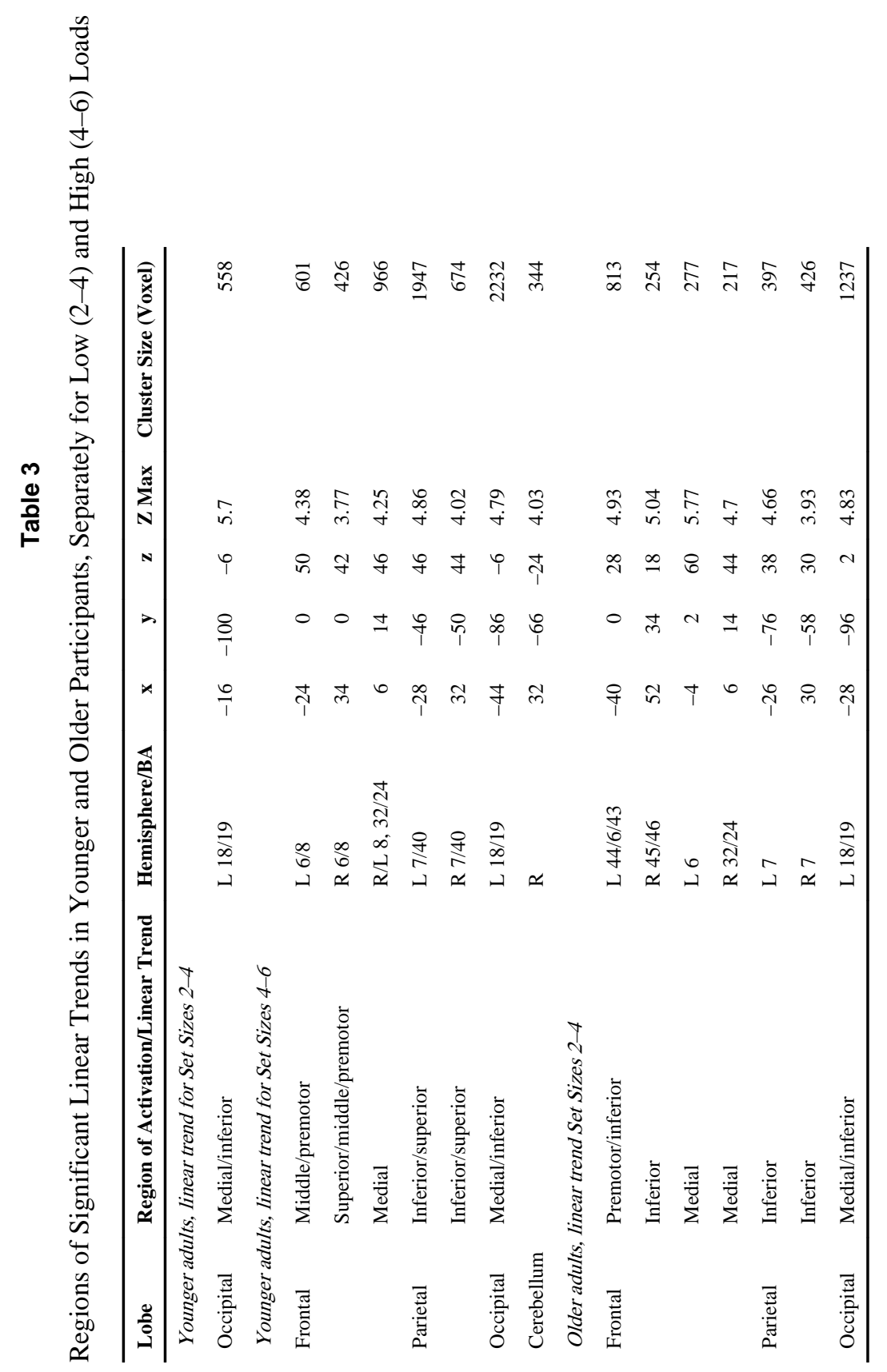




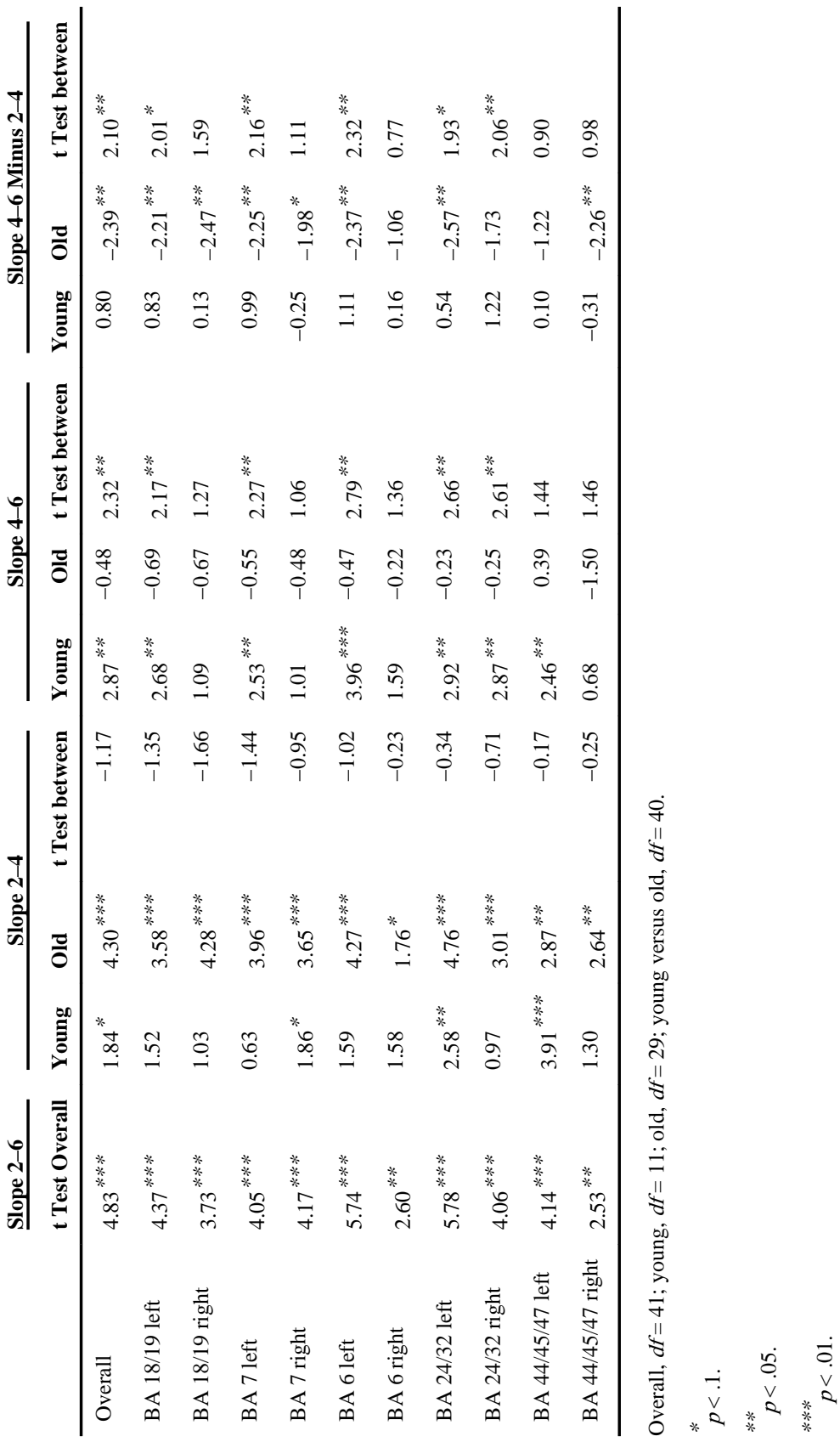

